\title{
Directional Auger Electron Spectroscopy - Possibilities, Limitations and Proposal of Improvements
}

\author{
S. MRÓZ AND Ł. ROK \\ Institute of Experimental Physics, University of Wrocław \\ pl. Maxa Borna 9, PL-50-204 Wrocław, Poland
}

Physical foundations of directional auger electron spectroscopy (DAES) and calculation of DAES profiles in single scattering cluster approach are presented. Limitations of this method (application only to investigation of the crystalline structure of homogeneous samples) is shown and explained as the result of participation of inelastically scattered electrons in the Auger signal generation. To extend the DAES application for the interface structure, the use of as low as possible energy of primary electrons is proposed because in such a case the participation of inelastically scattered electrons becomes negligible and single scattering cluster calculation should describe correctly the DAES profiles for interfaces. Besides, the extension of single scattering cluster calculations to the second elastic scattering is recommended. To check the technical possibility of DAES use in the proposed version, the Auger spectrum of $\mathrm{Cu} L M M$ peaks was recorded for a $\mathrm{Cu}_{3} \mathrm{Au}(001)$ sample with the use of a retarding field analyzer with the primary beam energy $1200 \mathrm{eV}$. Quality of this spectrum seems to be good enough for using in DAES.

PACS numbers: 61.05.J-, 68.35.Rh

\section{Description of DAES method and SSC approximation}

The influence of the primary electron beam direction on the Auger signals measured for crystalline samples is well recognized for many years (see, for example, reviews [1, 2] and references therein). Namely, distinct maxima of the measured Auger signal appear when the primary electron beam is parallel to the direction of some set of close packed rows of atoms in the crystal investigated. This opportunity was seen as the difficulty in a quantitative Auger analysis. On the other hand, it was used as a base of the method giving information about the crystalline structure of ultrathin layers and about the epitaxial relation between the deposited layer and its substrate (directional Auger electron spectroscopy - DAES in [3] and primary-beam diffraction-modulated electron emission - PDMEE in 
[4]). In this method, the Auger signal is collected by a large acceptance angle spectrometer RFA (retarding field analyzer) or CMA (cylindrical mirror analyzer) during the sample rotation around the axis lying in the sample surface plane, for the azimuth plane containing directions of close packed rows of atoms (Fig. 1). In such a way, polar DAES profiles are recorded. Such measurements are repeated for the primary electron beam energies contained between 1 and $2 \mathrm{keV}$. Positions of the maxima present in such polar profiles at the incidence angles common for all electron energies used (permanent maxima) correspond to the directions of close packed rows of atoms. Thus, positions of these maxima give information about the symmetry elements of the surface layer of investigated sample.

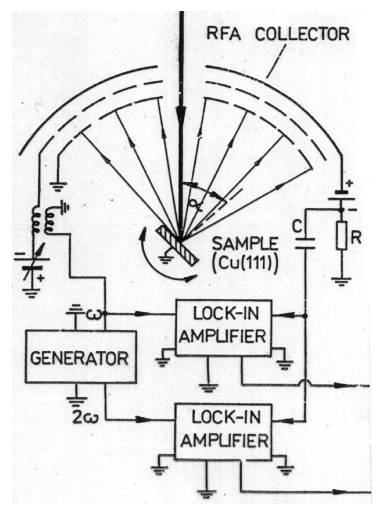

Fig. 1. DAES measuring system.

Theoretical description of DAES profiles based on the single scattering cluster (SSC) approximation for diffraction of the primary electron beam was proposed by Stuck et al. [5] with the use of formalism elaborated by Gao and Cao [6]. In this approximation (see Fig. 2), a wave function $\Psi\left(\boldsymbol{r}_{j}\right)$ at the $j$-th atom is the sum of the plane wave corresponding to the primary electron beam attenuated by inelastic scattering on its path to this atom and spherical waves elastically scattered (only once) on other atoms of the cluster and attenuated by inelastic scattering on their path to the $j$-th atom after this scattering

$$
\begin{aligned}
& \Psi\left(\boldsymbol{k}, \boldsymbol{r}_{j}\right) \sim \exp (\mathrm{i} \boldsymbol{k r})\left[\exp \left(-z_{j} / \lambda \cos \theta\right)+\sum_{i} \exp \left(-\mathrm{i} \boldsymbol{k} \boldsymbol{r}_{i j}\right) \exp \left(-z_{i} / \lambda \cos \theta\right)\right. \\
& \left.\left.\quad \times \exp \left(-r_{i j} / \lambda\right) f_{i}\left(\boldsymbol{k}, \boldsymbol{r}_{i j}, T\right) \exp \left(\mathrm{i} k r_{i j}\right) / k r_{i j}\right)\right],
\end{aligned}
$$

where $f_{j}$ is the dependent on the temperature $T$ scattering factor of the $j$-th atom, $\lambda$ is the inelastic mean free path for electrons used, $z_{i}$ is the depth of the $i$-th atom under the surface. The refraction of the primary beam at the cluster surface, which is indicated in Fig. 2, is taken into account. Namely, the sample inner potential is involved in determination of the wave vector $\boldsymbol{k}$ value and direction. 


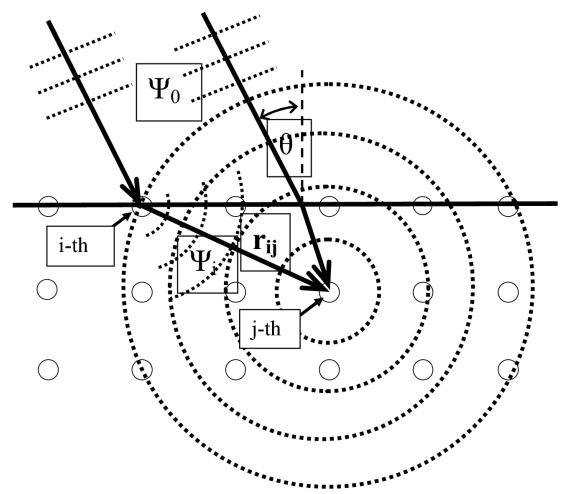

Fig. 2. Scheme of SSC calculations.

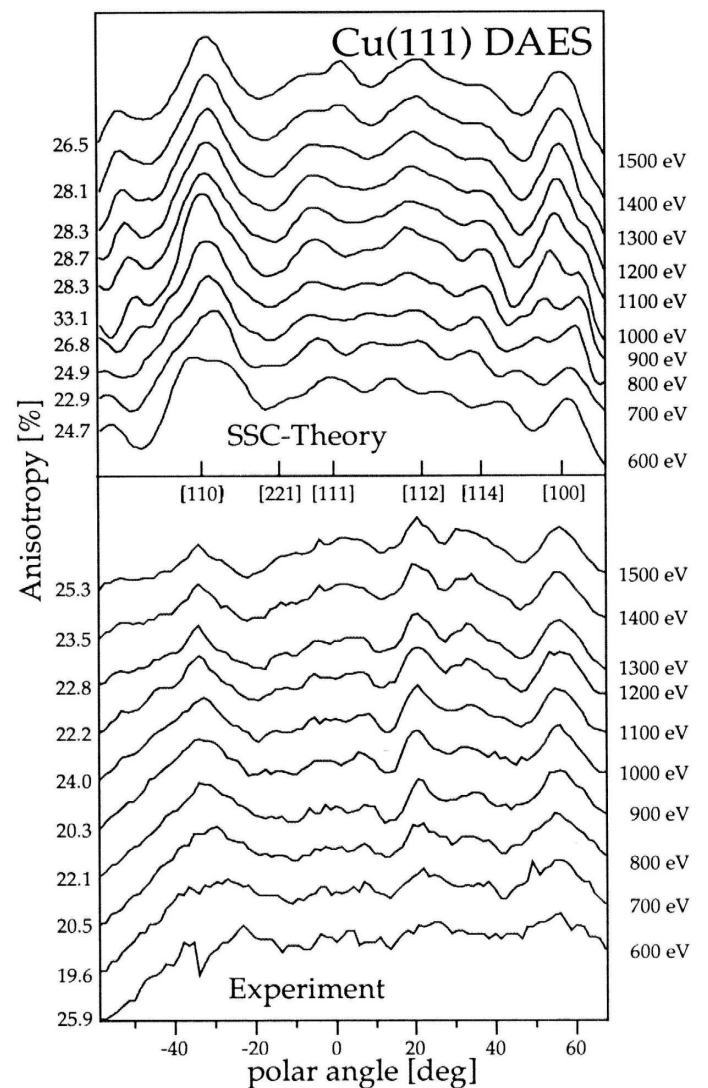

Fig. 3. Experimental DAES polar profiles for the $\mathrm{Cu} M_{2,3} V V$ Auger transition (62 eV), for $\mathrm{Cu}(111)$ face along the $[11 \overline{2}]-[1 \overline{1} 2]$ azimuth, compared with SSC calculations. The kinetic energy of the incidence electrons is indicated as well as the anisotropy [ $\left(I_{\max }-\right.$ $\left.\left.I_{\min }\right) / I_{\max }\right] 100 \%$ between $-40^{\circ}$ and $+60^{\circ}$. After [5]. 
For electron energies from several hundreds to several thousands eV, the forward focusing of electrons along the close packed rows of atoms results from this approximation. This focusing leads to the maximum values of $\mid \Psi\left(\boldsymbol{k},\left.\boldsymbol{r}_{j}\right|^{2}\right.$ and, as a consequence, to the maximum value of the Auger signal. Concerning the transport of the Auger electrons through the sample, their diffraction is neglected because of the large acceptance angle of the analyzer used. Additionally, it is supposed that the primary electrons backscattered inelastically in a deeper part of the sample do not excite the Auger electron emission from atoms in the surface layer with the thickness in the range of the inelastic mean free path of considered Auger electrons.

Thus, the calculated Auger signal $I(\boldsymbol{k})$ is

$$
I(\boldsymbol{k}) \sim \sum_{j}\left|\Psi\left(\boldsymbol{k}, \boldsymbol{r}_{j}\right)\right|^{2} A\left(z_{j} / \lambda_{\text {out }}\right)
$$

where an isotropic emission of the Auger electrons from an atom is supposed, and the geometry of the cluster/RFA entrance system and the damping of the Auger electrons in the cluster are taken into account in the $A$ factor.

SSC approximation was successfully used for description of DAES profiles measured for massive homogeneous crystal surfaces and for epitaxial homogeneous layers composed of several monolayers (see Fig. 3). For example, in [7] the (111) oriented silver multilayer film deposited on the $\mathrm{Cu}(001)$ face was found to be composed of four kinds of domains with different epitaxial orientation with respect to the substrate. What is more, the fitting of the calculated polar DAES profiles to the measured ones enabled to determine populations of particular orientations.

\section{Limitations and possible improvements of DAES}

The DAES method was proposed in [3] together with directional elastic peak electron spectroscopy (DEPES) where the signal of elastically scattered electrons is measured with the use of an RFA collector versus the primary beam incidence angle. Corresponding polar DAES and DEPES profiles for massive homogeneous samples as well as for multilayer films were found to be very similar (see, for example, Fig. 4). It was not a surprise because the diffraction of primary electrons is described in the SSC approach in the same way for DAES and DEPES, while the diffraction of elastically scattered electrons outgoing from the sample can be neglected as well as diffraction of the Auger electrons mentioned above. Because of this similarity, information about crystalline structure obtainable from DAES and DEPES profiles is the same in the case discussed above, while the measurement of DEPES profiles is much simpler and more exact than that for DAES profiles. On the other hand, the DAES method seemed to be attractive in investigation of surface alloys or ultrathin films deposited on a crystalline substrate. However, it has been found that for $0.8 \mathrm{ML}$ layer of silver on the $\mathrm{Cu}(001)$ face $[8,9]$, for $0.5 \mathrm{ML}$ of cobalt on the $\mathrm{Fe}(001)$ face [10] and for very thin layers of iron on the $(11 \overline{2} 0)$ face of cobalt [11], the DAES polar profiles for these layers recorded for 


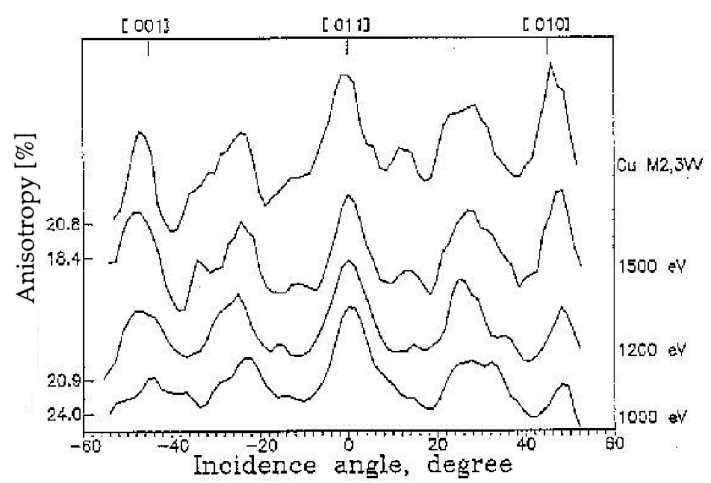

Fig. 4. DAES polar profile for the $\mathrm{Cu} M_{2,3} V V$ Auger transition $(62 \mathrm{eV})$ for the incidence electron energy of $1500 \mathrm{eV}$, and DEPES profiles for different energies for the

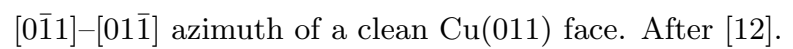

the primary electron energy $E=800 \mathrm{eV}$ or higher reproduce the shape of DEPES profiles of the substrate. On the other hand, for $E_{\mathrm{p}}=600 \mathrm{eV}$ the $\mathrm{Ag} M N N$ DAES profiles presented in $[8,9]$ are rather flat, without maxima characteristic of DEPES profiles. It should be pointed out that the energy $E=600 \mathrm{eV}$ is high enough for obtaining well pronounced maxima in DEPES and DAES profiles for the clean copper substrate and for thicker silver films.

These results can be explained as follows. In typical AES measurements the primary electron energy $E_{\mathrm{p}}$ is much higher than the ionization energy $E_{W}$ of an atom in the first step of the Auger process because the Auger signal shows maximum in such a case. However, not only the $\left|\Psi\left(\boldsymbol{r}_{j}\right)\right|^{2}$ mentioned above is responsible for the Auger process. This process is also initiated by the electrons backscattered inelastically in deeper layers of the sample and traveling to the sample surface. For homogeneous samples (amorphous or polycrystalline), the participation of backscattered electrons in generation of the Auger signals is taken into account with the use of so-called backscattering factor $B$ appearing in the formula for the Auger signal $I_{\alpha, W X Y}$ generated owing to $W X Y$ Auger transitions in atoms of an element $\alpha$ :

$$
I_{\alpha, W X Y} \sim \gamma_{\alpha, W X Y} \lambda \sigma_{\alpha, W}\left(E_{\mathrm{p}} / E_{W}\right) B\left(E_{\mathrm{p}} / E_{W}\right) I_{\mathrm{p}} n_{\alpha},
$$

where $\gamma$ is the probability of a $W X Y$ Auger transition in atoms of the element $\alpha$ with the ionized level $W$ (in competition with other possible $W X^{\prime} Y^{\prime}$ transitions and with $\mathrm{X}$-ray emission), $\lambda$ is the inelastic mean free path of considered Auger electrons in the sample investigated, $\sigma$ is the cross-section for the ionization of the $W$ level, $E_{\mathrm{p}}$ is the primary electron energy, $E_{W}$ is the ionization energy of the $W$ level, $I_{\mathrm{p}}$ is the current of primary electrons, and $n_{\alpha}$ is the concentration of $\alpha$ atoms in the sample.

Among the terms appearing in formula (3), $\sigma$ increases from zero at the ratio $E_{\mathrm{p}} / E_{W}=1$ to the maximum value for this ratio contained between 2 and 
3 and then decreases slowly whilst $B$ increases quickly from unity for this ratio increasing from unity to, say, three and next increases slower and slower. The measured Auger signal is also dependent on the direction of the primary electron beam and the direction from the sample to the Auger electron collector. Generally, it increases with the increase in the primary beam incidence angle (measured to the sample surface normal) because the average depth of the Auger electron generation decreases with this angle increase, which results in the decrease in the probability of inelastic scattering of the Auger electrons on their path to the sample surface. On the other hand, the increase in the emission angle of the Auger electrons increases their path to the sample surface and, as a consequence, decreases the measured Auger signal because of the increasing probability of inelastic scattering of the Auger electrons. Both dependences mentioned above are monotonic. For an Auger spectrometer based on the RFA analyzer with the large acceptance angle and with an axial electron gun used as a source of the primary electron beam, the dependence of the Auger signal on the incidence angle, changed by the sample rotation in relation to the RFA entrance, is quite weak (changes of this signal do not exceed a few percent for the incidence angle growing from zero to $60^{\circ}$ ).

Quite a different situation takes place for crystalline samples. Let us consider a crystalline sample composed of atoms $\alpha$ covered with a monolayer of atoms $\beta$ (see Fig. 5).

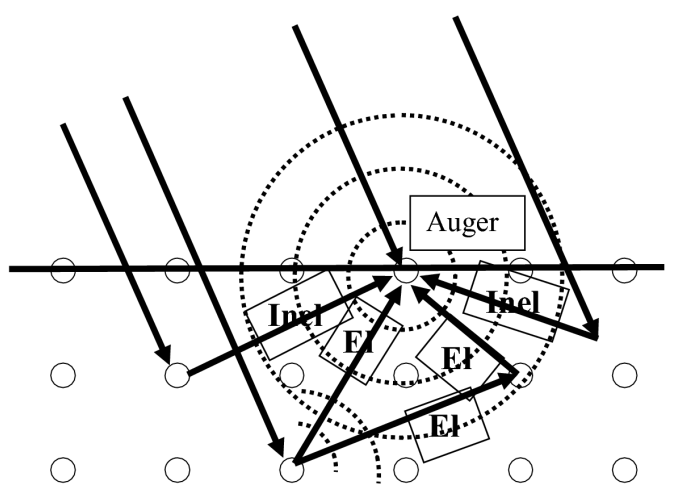

Fig. 5. Scheme of SSC calculation extended to the double scattering and inelastic scattering.

In such a case, the forward focusing of primary electrons does not concern even atoms $\beta$ belonging to close packed rows of atoms nonparallel to the sample surface because an atom $\beta$ can only be the first atom in such a row. Therefore $\Psi\left(\boldsymbol{r}_{j}\right)$ for such an atom, obtained using the SSC approach, should be the wave function of the primary electron beam $\Psi_{\mathrm{p}}\left(\boldsymbol{r}_{j}\right)$ with the addition of part $\Delta_{\mathrm{SSC}}\left(\boldsymbol{r}_{j}\right)$ originating from the elastic scattering of primary electrons for large angles of scattering on surrounding atoms 


$$
\Psi_{\mathrm{SSC}}\left(\boldsymbol{r}_{j}\right)=\Psi_{\mathrm{p}}\left(\boldsymbol{r}_{j}\right)+\Delta_{\mathrm{SSC}}\left(\boldsymbol{r}_{j}\right) .
$$

This addition is expected to be small, if we have in mind that the intensity of the elastically scattered electron stream directed into the RFA analyzer acceptance angle is only a few percent of the primary beam intensity. Thus, DAES profiles for atoms $\beta$ should be rather flat, without distinct maxima. However, the SSC approach describes only the elastic scattering and only the first scattering is taken into account. On the other hand, for crystalline samples the backscattering factor $B$ appearing in the formula (3) should be sensitive to the direction of the primary electron beam with respect to the directions of close packed rows of atoms. Namely, the shape of polar profiles for inelastically scattered electrons is, for the energy losses $\Delta E$ not exceeding about $300 \mathrm{eV}$ (see Fig. 6), similar to the corresponding DEPES profiles $[2,8]$. For this reason, the backscattering factor becomes maximal

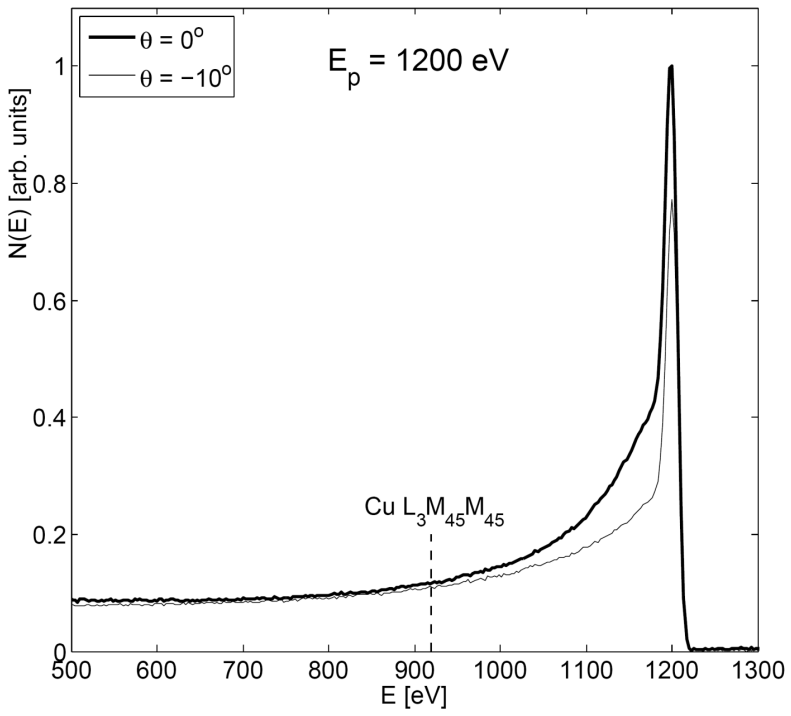

Fig. 6. Energy distribution of electrons scattered from the $\mathrm{Cu}_{3} \mathrm{Au}(001)$ sample for the incidence electron energy $E_{\mathrm{p}}=1200 \mathrm{eV}$, for the incidence angle $\theta=0^{\circ}$ and $-10^{\circ}$.

for primary electron beam directions parallel to one of the close packed rows of atoms and clearly visible maxima are present even in DAES profiles measured for the monolayer of adsorbate atoms deposited on the surface of crystalline sample. On the other hand, such maxima do not appear in the corresponding profiles calculated in the SSC approach. To take this into account, relation (2) should be changed as follows:

$$
I(\boldsymbol{k})=I_{\mathrm{el}}(\boldsymbol{k})+I_{\text {inel }}(\boldsymbol{k}),
$$

where $I_{\mathrm{el}}(\boldsymbol{k})$ is introduced instead of $I(\boldsymbol{k})$ present in Eq. (2). Thus the DAES method in the version used so far is rather useless for investigation of interface atomic structure. 
The way of elimination of limitations and problems mentioned above seems to be a drastic decrease in the ratio $E_{\mathrm{p}} / E_{W}$ (see relation (1)). Of course, the decrease in ratio $E_{\mathrm{p}} / E_{W}$ close to the unity leads to a strong decrease in the cross-section $\sigma_{\alpha, W}\left(E_{\mathrm{p}} / E_{W}\right)$ of the ionization of the level $W$ and, as a consequence, to a strong decrease in the measured Auger signal, but the participation of inelastically scattered electrons in creation of an Auger signal will be decreased much stronger and relation (2) becomes a much better approximation. However, the Auger transitions with the energy of the Auger electrons contained between several hundreds and several thousands eV should be chosen because the SSC approach can be successfully used for electrons from this energy range.

To check the possibility of DAES measurement in the version proposed above, the $\mathrm{Cu} L M M$ Auger peaks (at 776, 849, and $920 \mathrm{eV}$ ) were recorded for the $\mathrm{Cu}_{3} \mathrm{Au}(001)$ sample, in the $\mathrm{d} N(E) / \mathrm{d} E$ mode, with the use of an RFA analyzer, for $E_{\mathrm{p}}=1200 \mathrm{eV}\left(E_{\mathrm{p}} / E_{W} \approx 1.28\right)$. The recorded spectrum is presented in Fig. 7. This result is encouraging but the effectiveness of the method proposed should be checked for a homogeneous sample with a monolayer of other atoms deposited on its surface.

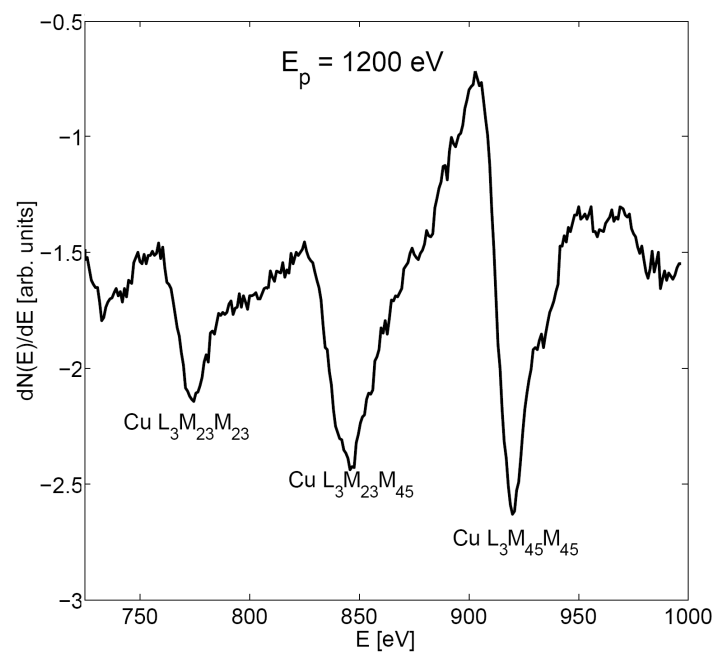

Fig. 7. $\mathrm{Cu} L V V$ Auger spectrum in the $\mathrm{d} N(E) / \mathrm{d} E$ mode recorded using an RFA analyzer for the incidence electron energy $E_{\mathrm{p}}=1200 \mathrm{eV}$ and for incidence angle $\theta=0^{\circ}$.

It should be added here that the SSC approach is not exact because the second, third and so on elastic scatterings are not taken into account in it. Thus, the part $I_{\mathrm{el}}(\boldsymbol{k})$ in relation (6) does not describe the participation of all elastically scattered primary electrons in generation of the Auger signal. Further improvement in calculation of expected DAES profiles can be reached with the use of multiple scattering cluster (MSC) approach. However, such an approach is much more time consuming and the effectiveness of its application should be tested in practice. 


\section{References}

[1] S. Mróz, Surf. Rev. Lett. 4, 117 (1997).

[2] S. Valeri, A. di Bona, Surf. Rev. Lett. 4, 141 (1997).

[3] S. Mróz, M. Nowicki, Surf. Sci. 297, 66 (1993).

[4] S. Valeri, A. di Bona, G.C. Gazzadi, Surf. Interface Anal. 21, 852 (1994).

[5] A. Stuck, M. Nowicki, S. Mroz, D. Naumovic, J. Osterwalder, Surf. Sci. 306, 21 (1994).

[6] Y. Gao, Jianming Cao, Phys. Rev. B 43, 11460 (1991).

[7] M. Nowicki, J. Osterwalder, Surf. Sci. 408, 165 (1998).

[8] S. Mróz, A. Mróz, M. Nowicki, J. Electron. Spectrosc. Relat. Phenom. 76, 635 (1995).

[9] M. Nowicki, P. Krupa, Vacuum 48, 313 (1997).

[10] A. di Bona, P. Luches, A. Borghi, F. Rossi, S. Valeri, Surf. Rev. Lett. 6, 599 (1999).

[11] A. Borghi, A. di Bona, P. Luches, S. Valeri, Surf. Sci. 466, 30 (2000).

[12] M. Nowicki, S. Mróz, Vacuum 46, 537 (1995). 